\title{
Rapid progression of disease from immunotherapy following targeted therapy: insights into treatment management and sequence
}

\author{
Rebecca Pharaon, Isa Mambetsariev, Jeremy Fricke, Ravi Salgia \\ Department of Medical Oncology and Therapeutics Research, City of Hope National Medical Center, Duarte, CA, USA \\ Contributions: (I) Conception and design: R Pharaon, I Mambetsariev, R Salgia; (II) Administrative support: R Pharaon, I Mambetsariev, R Salgia; \\ (III) Provision of study materials or patients: R Pharaon, J Fricke, R Salgia; (IV) Collection and assembly of data: R Pharaon, J Fricke, R Salgia; (V) \\ Data analysis and interpretation: R Pharaon, I Mambetsariev, R Salgia; (VI) Manuscript writing: All authors; (VII) Final approval of manuscript: All \\ authors. \\ Correspondence to: Dr. Ravi Salgia. Department of Medical Oncology and Therapeutics Research, City of Hope National Medical Center, Building 51, \\ 1500 W Duarte Road, Duarte, California 91010, USA. Email: rsalgia@coh.org.
}

\begin{abstract}
With emerging promising therapeutic regimens in non-small cell lung cancer (NSCLC), the standard-of-care treatments for a variety of histologic and mutated subgroups in NSCLC has been regularly shifting in response to landmark clinical trials. However, with the availability of a range of therapeutic agents, clear grouping of patient populations to appropriate treatment strategies is essential. In this review, we illustrate past and current treatment strategies in NSCLC, specifically focusing on targeted therapy and immunotherapy. We describe a complex clinical scenario that oncologists will encounter of patients with multiple actionable mutations such as epidermal growth factor receptor (EGFR) sensitizing mutations and high expression of programmed death-ligand 1 (PD-L1). Recent data regarding sequential therapy of EGFR tyrosine kinase inhibitors (TKIs) and immune checkpoint inhibitors (ICIs) demonstrate severe adverse interactions between the therapies that impact patient quality-of-life and outcomes. As we enter further into an era of personalized and precision medicine, guidelines and standard-of-care therapies are essential to define separate patient groups based on molecular testing, histology, comorbidities, and more. This article explores the current status of generally understudied patient groups in NSCLC and proposes future directions in therapeutic strategies.
\end{abstract}

Keywords: Non-small cell lung cancer (NSCLC); EGFR tyrosine kinase inhibitors (TKIs); immunotherapy; next-generation sequencing (NGS); rapid progression

Submitted Jul 11, 2019. Accepted for publication Jul 29, 2019.

doi: $10.21037 /$ jtd.2019.08.14

View this article at: http://dx.doi.org/10.21037/jtd.2019.08.14

\section{Introduction}

Lung cancer continues to be one of the most common forms of cancer with an estimated incidence of 228,150 new cases and 142,670 deaths in the U.S. in 2019 (1). The landscape of treatment for metastatic non-small cell lung cancer (NSCLC) has exponentially grown in the past decade with prominent roles for personalized treatment with targeted therapy and immunotherapy. These advances are ideal in simple clinical scenarios where next-generation sequencing (NGS) testing reports a single sensitizing actionable mutation in patients. However, treatment strategies are more complex in cases where patients have multiple cancer-driving actionable mutations that are gained and lost over the course of cancer treatment. Within this subset of patients who display an actionable mutation such as epidermal growth factor receptor (EGFR) as well as high programmed death ligand-1 (PD-L1) expression, standard therapeutic options are unclear. Moreover, the sequence 
of treatment regimens is another significant component to consider in these patients to avoid adverse interactions and rapid progression of disease. Long-term treatment strategies in patients with synchronous EGFR mutations and high PD-L1 expression remain largely undefined but ongoing and future studies continue to develop in this field. This review will explore the roles of EGFR tyrosine kinase inhibitors (TKIs) and immune checkpoint inhibitors (ICIs) in the current climate of lung cancer, antagonistic effects of these two therapies in patients whose cancer expresses both mutations, and future directions for this population of patients in NSCLC.

\section{EGFR TKIs and immunotherapy}

EGFR mutations are found in roughly $13 \%$ to $22 \%$ of patients and have become a significant therapeutic target in NSCLC (2). There exist various generations of drugs that inhibit the EGFR tyrosine kinase domain and research has established their superiority over chemotherapy in EGFR-mutated NSCLC patients $(3,4)$. Within the past 2 years, the phase III FLAURA trial compared the efficacy of EGFR TKIs and discovered that osimertinib, a thirdgeneration irreversible EGFR TKI, was associated with significantly superior progression-free survival and fewer grade 3 or higher adverse events (AEs) compared to various first and second-generation TKIs like erlotinib and gefitinib (5). This landmark study transformed standardof-care treatment in EGFR-mutated lung cancer with the approval of osimertinib as first-line treatment. This is especially significant in light of increasing importance of ICIs in NSCLC.

Interest in immunotherapy has drastically grown over the years across all cancer types (6-9). Multiple trials have demonstrated the superiority of immunotherapy over chemotherapy in terms of overall survival and progressionfree survival in previously treated, metastatic NSCLC (10-13). More importantly, two groundbreaking trials, KEYNOTE-024 and KEYNOTE-042, reported efficacy of first-line treatment with pembrolizumab plus chemotherapy and pembrolizumab monotherapy in metastatic NSCLC patients depending on PD-L1 expression by tumor proportion score (TPS) $(14,15)$. Patients undergoing treatment with anti-programmed cell death-1 (PD-1) or anti-PD-L1 antibodies report better quality-of-life and less toxicities that are associated with chemotherapy (16).

Multiple subgroup analyses of patients with both EGFR sensitizing mutations and high PD-L1 expression were conducted retrospectively in numerous breakthrough trials and indicated poor response rates to immunotherapy (17-19). This was further evaluated in several retrospective studies evaluating response rates in EGFR-mutated patient populations and found that immunotherapy was associated with failed treatment response $(20,21)$. A recent phase II study in EGFR-mutated NSCLC patients with no prior EGFR TKI treatment underwent treatment with pembrolizumab to guide treatment strategies (22). The investigators wanted to examine possible ICI efficacy and the results demonstrated no response utility from pembrolizumab (22).

Notably, a significant retrospective study was recently reported regarding the significance of therapy sequence on immune-related adverse event (irAE) incidence in a subset of patients (23). The study identified 126 NSCLC patients with an EGFR mutation who were treated with ICIs and EGFR TKIs. Patients who underwent treatment with immunotherapy followed by osimertinib were correlated with severe irAEs but this correlation was absent in patients who underwent EGFR TKI treatment then immunotherapy. This incidence was not noted in patients treated with any other EGFR TKIs and ICIs. In current treatment standards, osimertinib is the superior first-line treatment in EGFRmutated NSCLC. This study is especially relevant in today's climate with the high rates of osimertinib use and PD-1 or PD-L1 blockade in metastatic NSCLC.

\section{Complex clinical scenario}

A 55-year-old male with no smoking history but reported occupational inhalational exposure presented with a chronic cough, scantily production of phlegm, and considerable dyspnea. A chest X-ray was performed showing multiple bilateral pulmonary nodules and a right lung mass. Further workup included a chest computed tomography (CT) that demonstrated a $2.7 \mathrm{~cm} \times 2.6 \mathrm{~cm}$ right upper lobe lung mass, multiple bilateral pulmonary nodules, subcarinal, supraclavicular, and paratracheal adenopathy, and multiple bone metastases. Pathology from the CT-guided biopsy of the right upper lobe mass revealed moderately differentiated adenocarcinoma with predominant micropapillary pattern, clinical stage IVB, cT1cN3M1b. Molecular testing by immunohistochemistry (IHC) revealed an EGFR exon 19 deletion, PD-L1 (22C3) expression of 95\%, and PD-1 positive in $50 \%$ of tumor infiltrating lymphocytes. The patient was originally planned to enroll in a clinical trial but due to worsening left posterior chest wall pain and 
shortness of breath, he was started on erlotinib $150 \mathrm{mg}$ per day. Restaging imaging showed a good response to erlotinib but due to grade 2 rash as well as severe fatigue, the dose was reduced to $100 \mathrm{mg}$ po daily. Guardant 360 liquid biopsy was ordered in the meantime but reported no mutations. Patient had a continued response to treatment and erlotinib was dose reduced again to $75 \mathrm{mg}$ daily due to side effects.

Unfortunately, 11 months after starting erlotinib, restaging chest CT demonstrated progression of disease in the lungs and bones and a biopsy of the lung mass as well as NGS testing was ordered. From the biopsy specimen, Foundation Medicine reported EGFR exon 19 deletion, amplification, and the presence of T790M, a known mechanism of resistance to EGFR TKIs (24). The report also found KIT I571L, AKT2, ARFRP1, CCNE1, GNAS, and MCL1 amplification, TP53 R213L mutations, and PD-L1 high expression of 90\%. Liquid biopsy testing demonstrated similar results with the detection of EGFR exon 19 deletion, T790M, KIT I571L and a FGFR2 G817S mutation. Due to the presence of EGFR T790M mutation, the patient was subsequently started on osimertinib with a continued response noted on restaging imaging for the duration of 10 months until a CT chest, abdomen, pelvis scan showed slightly enlarged lung nodules. A PET scan and a liquid biopsy were requested to confirm true progression of disease, but showed overall continued response and decreased tumor burden. Liquid biopsy results only identified EGFR exon 19 deletion mutation. Thus, the patient continued with osimertinib until 3 months later where restaging imaging demonstrated true progression of disease in bilateral pulmonary nodules and lymph nodes.

Once again, a biopsy of one of the enlarging nodules, tissue and liquid NGS, and PD-L1 IHC testing were requested. Liquid biopsy detected EGFR exon 19 deletion, but no presence of T790M mutation. It also discovered an ERBB2 exon 20 insertion (Y772_A775dup) and TP53 R213L mutations with a PD-L1 expression of $70 \%$ on the biopsy specimen. Our institutional NGS testing on the biopsy specimen reported EGFR exon 19 deletion, MET, TERT, AKT2, and CCNE1 amplification, TP53 R213L, and $98 \%$ PD-L1 expression. Due to the high expression of PD-L1, his treatment was switched to carboplatin, pemetrexed, and pembrolizumab. Pembrolizumab was eventually held during the third cycle due to fatigue. During treatment, the patient was hospitalized for shortness of breath and was found to have pulmonary emboli in the lungs, treated with enoxaparin sodium. After three cycles of the regimen, a CT scan unfortunately demonstrated rapid progression of disease and the patient was immediately started on docetaxel and ramucirumab. Although the patient exhibited a partial response to treatment after two cycles, it was discontinued due to hospitalization for sepsis with hypotension and tachycardia. We considered gemcitabine or dacomitinib, a selective and irreversible inhibitor of EGFR, but decided to move forward with dacomitinib due to loss of EGFR T790M on previous NGS testing. The patient tolerated dacomitinib well but after 3 months on treatment, restaging imaging demonstrated progression of disease in the lungs, lymph nodes, and new bilateral adrenal nodules suspicious for metastases. Liquid biopsy at that time reported a reemergence of EGFR T790M as well as a BRCA2 K437fs mutation with other consistent mutations seen from previous NGS reports including EGFR exon 19 deletion, ERBB2 exon 20 insertion, TP53 R213C, and CCNE1 amplification.

It was then decided that the patient should re-start docetaxel and ramucirumab at a reduced dose since he previously responded to the regimen although it was not well tolerated. Unfortunately, the patient developed confusion, difficulty speaking, and forgetfulness in the meantime. An MRI of the brain was ordered and demonstrated innumerable intracranial metastases. He was then admitted to start whole brain radiation therapy for a total dose of 24 Gray over 8 fractions. The patient tolerated the treatment well but his general condition quickly deteriorated. He was transferred to hospice care and subsequently passed away. Treatment history is summarized and visualized in Figure 1.

\section{Discussion and future directions}

With the increase of interest in immunotherapy across cancer types, many questions remain about long term side effects, its role in cancers with actionable mutations, and more. Recent published data, including a phase II trial of pembrolizumab in TKI naïve NSCLC patients with an EGFR mutation and subgroup analyses from multiple landmark phase III trials, demonstrate a lack of response in EGFR-mutated cancers with monotherapy ICIs $(10,17,18,20,22)$. However, proven treatment strategies still remain unclear.

In this complex clinical case, the patient underwent multiple lines of therapy starting with two EGFR TKIs with which he exhibited a prolonged response for an average of 12 months until he progressed. Following erlotinib and osimertinib therapy, the patient was placed on 


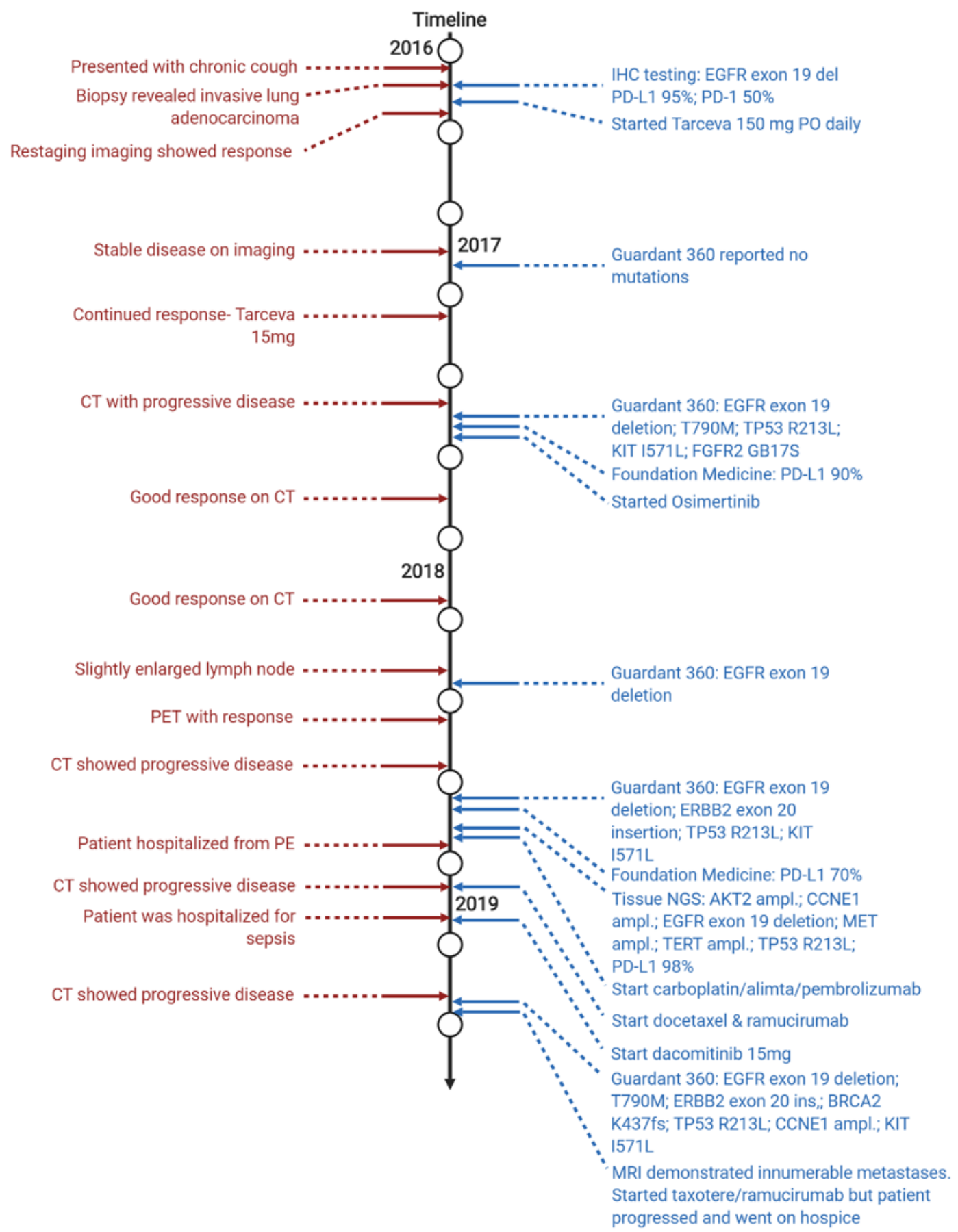

Figure 1 Clinical, radiographic, and molecular events throughout treatment history. The left column timeline displays clinical and radiographic information while the right column timeline indicates treatment regimens and molecular testing data (each line between circles represents 3 months). 

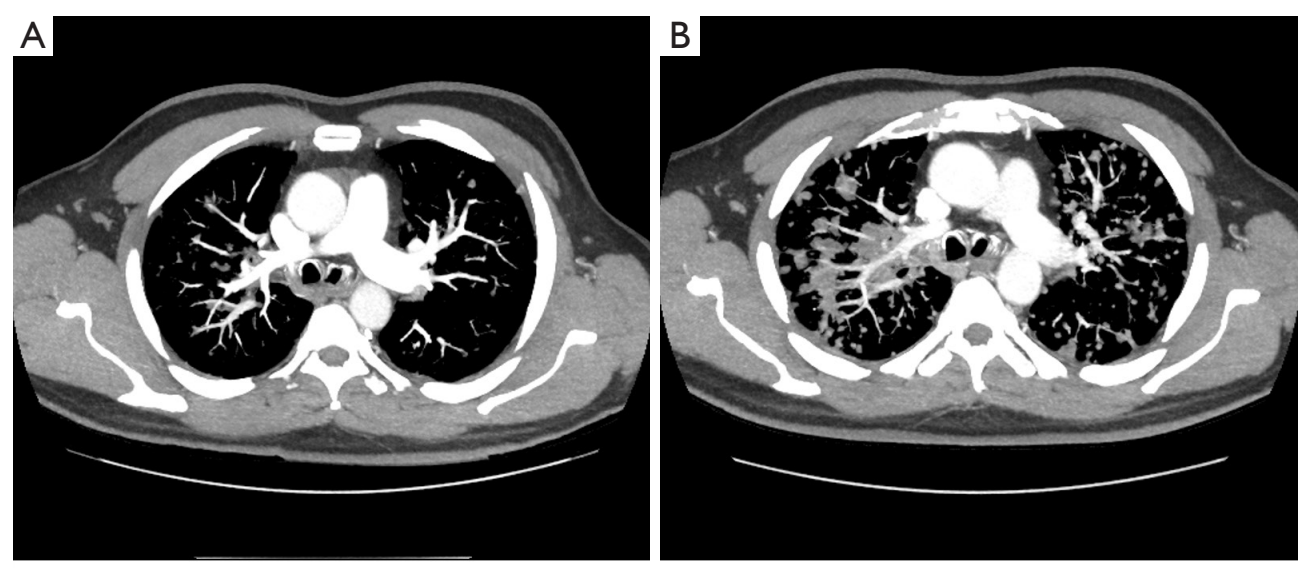

C

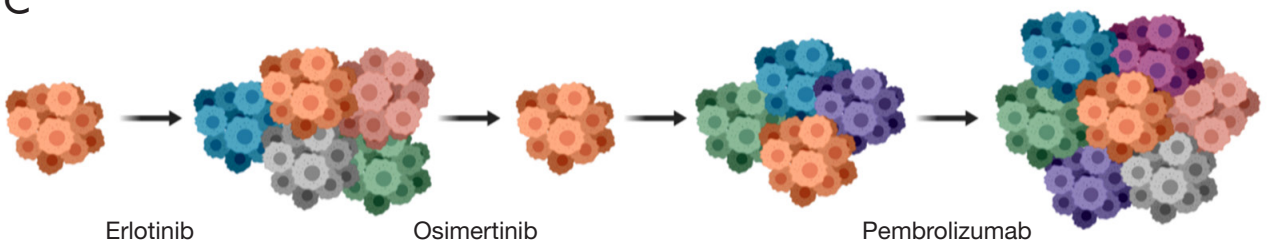

Figure 2 Rapid progression of disease on immunotherapy following osimertinib treatment. (A) CT chest scan showing progression of disease in the lungs after 13 months on osimertinib treatment; (B) CT chest scan showing rapid progression of disease in the lungs 3 months after discontinuing osimertinib and after 3 cycles of carboplatin, pemetrexed, and pembrolizumab; (C) representation of tumor heterogeneity and burden throughout treatment. (A,B) Axial CT scanning. CT, computed tomography.

carboplatin, pemetrexed, and pembrolizumab. Although the patient's PD-L1 was high, pembrolizumab monotherapy was not considered due to poor response in EGFR- and ERBB2-mutated NSCLC $(17,25)$. Pembrolizumab was not well tolerated and he quickly progressed after 3 cycles of combination cytotoxic chemotherapy and immunotherapy. The average duration of response was significantly higher with the EGFR TKIs versus the combination regimen of ICIs and chemotherapy. On the other hand, Kunimasa et al. published a case report of a patient diagnosed with metastatic lung adenocarcinoma who was found to have an EGFR exon 19 deletion and high PD-L1 expression, similar to our patient (26). Unlike our patient, the authors reported that their patient experienced rapid progression of disease to erlotinib but managed an almost complete response to anti-PD-1 monoclonal antibody pembrolizumab. This discrepancy needs to be addressed in a validated clinical trial setting.

Treatment regimen order has become increasingly important as seen with the recent retrospective analysis on irAEs from treatment with ICIs and EGFR TKI osimertinib (23). Severe AEs were observed in patients who received ICI therapy followed by osimertinib but none were reported if the treatment order was reversed (23). The patient described above received osimertinib initially but due to progression of disease was switched to carboplatin, pemetrexed, pembrolizumab and was hospitalized after the first cycle from pulmonary emboli. This event is attributed to disease progression and subsequent restaging imaging after three cycles confirmed rapid disease progression and increased tumor burden when compared to baseline CT chest imaging from 3 months prior (Figure 2). Our patient experienced no irAEs, severe or otherwise, however he experienced acute disease progression following treatment with osimertinib then ICIs. This lack of response is consistent with reported low response rates in patients with parallel molecular mutations (17). However, even the addition of chemotherapy to pembrolizumab did not promote response efficacy in this patient. Interestingly, after this regimen, the patient exhibited a partial response to chemotherapy agent docetaxel and antiangiogenic vascular endothelial growth factor receptor (VEGFR)-2 inhibitor, suggesting the utility of chemotherapy and other inhibitors in later treatment lines in patients with multiple driving mutations. Further research is necessary to correctly classify patient populations that would be affected by rapid 


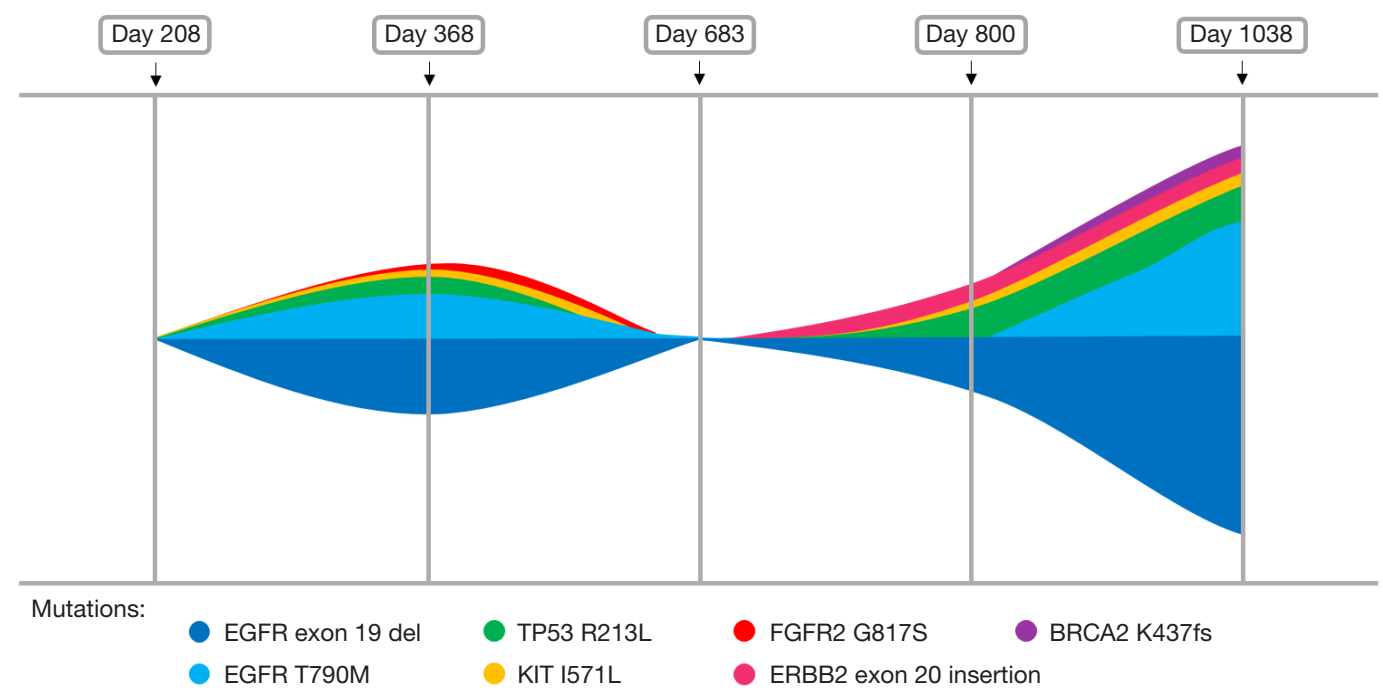

Figure 3 Evolution of reported mutations via liquid biopsy NGS testing throughout treatments. NGS, next-generation sequencing.

progression from sequential TKI and immunotherapy treatments.

Consistent molecular testing via liquid or tissue biopsies and routine restaging imaging play a significant role in decided treatment choice and monitoring response in patients. Serial liquid and tissue biopsies were necessary in detecting mutations of resistance as well as tumor heterogeneity and clonal evolution (Figures 2C,3). NSCLC is a complex disease and the additional of temporal molecular sequencing of blood and specimen was vital in observing sub-clonal populations, emergence and disappearance of resistance mutations, and tumor heterogeneity (27).

\section{Conclusions}

Despite many advances in the treatment of metastatic NSCLC, treatment approaches or combinations for several cohorts of patients remain ambiguous. We demonstrated a complex clinical case of a patient reported to have EGFRmutated metastatic lung adenocarcinoma and high PDL1 expression who underwent multiple lines of therapy including three different EGFR TKIs, chemotherapy, and immunotherapy. This clinical scenario revealed that EGFR TKIs were associated with a longer duration of progression free survival while the treatment combination with immunotherapy resulted in rapid progression of disease. However, additional studies of patients who have synchronous cancer-driving mutations is needed to confirm the utility and sequence of various agents. There was no documentation of severe irAEs that are seen in patients who undergo sequential PD-1 blockade followed by osimertinib EGFR TKI treatment, consistent with results reported in the retrospective study by Schoenfeld et al. (23). Further analysis and evaluation is necessary to clearly define therapeutic options and sequence for this subset of patients.

\section{Acknowledgments}

The authors would like to thank City of Hope nurses and supportive staff for their dedication to their patients.

Funding: This work was supported by the National Cancer Institute of the National Institutes of Health under award numbers P30CA033572, U54CA209978, and R01CA218545.

\section{Footnote}

Provenance and Peer Review: This article was commissioned by the Guest Editor (Ammar Chaudhry) for the series "Role of Precision Imaging in Thoracic Disease" published in Fournal of Thoracic Disease. The article was sent for external peer review organized by the Guest Editor and the editorial office.

Conflicts of Interest: All authors have completed the ICMJE uniform disclosure form (available at http://dx.doi. org/10.21037/jtd.2019.08.14). The series "Role of Precision Imaging in Thoracic Disease" was commissioned by the 
editorial office without any funding or sponsorship. The authors have no other conflicts of interest to declare.

Ethical Statement: The authors are accountable for all aspects of the work in ensuring that questions related to the accuracy or integrity of any part of the work are appropriately investigated and resolved.

Open Access Statement: This is an Open Access article distributed in accordance with the Creative Commons Attribution-NonCommercial-NoDerivs 4.0 International License (CC BY-NC-ND 4.0), which permits the noncommercial replication and distribution of the article with the strict proviso that no changes or edits are made and the original work is properly cited (including links to both the formal publication through the relevant DOI and the license). See: https://creativecommons.org/licenses/by-nc-nd/4.0/.

\section{References}

1. Siegel RL, Miller KD, Jemal A. Cancer statistics, 2019. CA: A Cancer Journal for Clinicians 2019;69:7-34.

2. Antonoff $M B, D^{\prime}$ Cunha J. Non-small cell lung cancer: the era of targeted therapy. Lung Cancer (Auckl) 2012;3:31-41.

3. Rosell R, Carcereny E, Gervais R, et al. Erlotinib versus standard chemotherapy as first-line treatment for European patients with advanced EGFR mutation-positive non-small-cell lung cancer (EURTAC): a multicentre, open-label, randomised phase 3 trial. Lancet Oncol 2012;13:239-46.

4. Mok TS, Wu YL, Ahn MJ, et al. Osimertinib or PlatinumPemetrexed in EGFR T790M-Positive Lung Cancer. N Engl J Med 2017;376:629-40.

5. Soria JC, Ohe Y, Vansteenkiste J, et al. Osimertinib in Untreated EGFR-Mutated Advanced Non-Small-Cell Lung Cancer. N Engl J Med 2018;378:113-25.

6. Robert C, Schachter J, Long GV, et al. Pembrolizumab versus Ipilimumab in Advanced Melanoma. $\mathrm{N}$ Engl J Med 2015;372:2521-32.

7. Seiwert TY, Burtness B, Mehra R, et al. Safety and clinical activity of pembrolizumab for treatment of recurrent or metastatic squamous cell carcinoma of the head and neck (KEYNOTE-012): an open-label, multicentre, phase 1b trial. Lancet Oncol 2016;17:956-65.

8. Bellmunt J, de Wit R, Vaughn DJ, et al. Pembrolizumab as Second-Line Therapy for Advanced Urothelial Carcinoma. N Engl J Med 2017;376:1015-26.
9. Kaufman HL, Russell J, Hamid O, et al. Avelumab in patients with chemotherapy-refractory metastatic Merkel cell carcinoma: a multicentre, single-group, open-label, phase 2 trial. Lancet Oncol 2016;17:1374-85.

10. Borghaei H, Paz-Ares L, Horn L, et al. Nivolumab versus Docetaxel in advanced nonsquamous non-small-cell lung cancer. N Engl J Med 2015;373:1627-39.

11. Herbst RS, Baas P, Kim DW, et al. Pembrolizumab versus docetaxel for previously treated, PD-L1-positive, advanced non-small-cell lung cancer (KEYNOTE-010): a randomised controlled trial. Lancet 2016;387:1540-50.

12. Reck M, Rodríguez-Abreu D, Robinson AG, et al. Pembrolizumab versus Chemotherapy for PD-L1Positive Non-Small-Cell Lung Cancer. N Engl J Med 2016;375:1823-33.

13. Rittmeyer A, Barlesi F, Waterkamp D, et al. Atezolizumab versus docetaxel in patients with previously treated non-small-cell lung cancer (OAK): a phase 3, openlabel, multicentre randomised controlled trial. Lancet 2017;389:255-65.

14. Mok TSK, Wu YL, Kudaba I, et al. Pembrolizumab versus chemotherapy for previously untreated, PD-L1expressing, locally advanced or metastatic non-small-cell lung cancer (KEYNOTE-042): a randomised, open-label, controlled, phase 3 trial. Lancet 2019;393:1819-30.

15. Gandhi L, Rodriguez-Abreu D, Gadgeel S, et al. Pembrolizumab plus chemotherapy in metastatic nonsmall-cell lung cancer. N Engl J Med 2018;378:2078-92.

16. Brahmer JR, Rodríguez-Abreu D, Robinson AG, et al. Health-related quality-of-life results for pembrolizumab versus chemotherapy in advanced, PD-L1-positive NSCLC (KEYNOTE-024): a multicentre, international, randomised, open-label phase 3 trial. Lancet Oncol 2017;18:1600-9.

17. Gainor JF, Shaw AT, Sequist LV, et al. EGFR Mutations and ALK Rearrangements Are Associated with Low Response Rates to PD-1 Pathway Blockade in Non-Small Cell Lung Cancer: A Retrospective Analysis. Clin Cancer Res 2016;22:4585-93.

18. Lee CK, Man J, Lord S, et al. Clinical and Molecular Characteristics Associated With Survival Among Patients Treated With Checkpoint Inhibitors for Advanced NonSmall Cell Lung Carcinoma: A Systematic Review and Meta-analysis. JAMA Oncol 2018;4:210-6.

19. Lee CK, Man J, Lord S, et al. Checkpoint inhibitors in metastatic EGFR-mutated non-small cell lung cancer-a meta-analysis. J Thorac Oncol 2017;12:403-7.

20. Lisberg A, Garon EB. Lack of clearly defined role for anti- 
programmed death-(ligand) 1 therapy in epidermal growth factor receptor mutated non-small cell lung cancer. Transl Lung Cancer Res 2019;8:195-7.

21. Hastings K, Yu HA, Wei W, et al. EGFR mutation subtypes and response to immune checkpoint blockade treatment in non-small-cell lung cancer. Ann Oncol 2019;30:1311-20.

22. Lisberg A, Cummings A, Goldman JW, et al. A Phase II Study of Pembrolizumab in EGFR-Mutant, PD-L1+, Tyrosine Kinase Inhibitor Naive Patients With Advanced NSCLC. J Thorac Oncol 2018;13:1138-45.

23. Schoenfeld AJ, Arbour KC, Rizvi H, et al. Severe immune-related adverse events are common with sequential PD-(L)1 blockade and osimertinib. Ann Oncol 2019;30:839-44.

Cite this article as: Pharaon R, Mambetsariev I, Fricke J, Salgia R. Rapid progression of disease from immunotherapy following targeted therapy: insights into treatment management and sequence. J Thorac Dis 2020;12(9):5096-5103. doi: 10.21037/jtd.2019.08.14
24. Kobayashi S, Boggon TJ, Dayaram T, et al. EGFR Mutation and Resistance of Non-Small-Cell Lung Cancer to Gefitinib. N Engl J Med 2005;352:786-92.

25. Yu S, Liu D, Shen B, et al. Immunotherapy strategy of EGFR mutant lung cancer. Am J Cancer Res 2018;8:2106-15.

26. Kunimasa K, Nakamura H, Sakai K, et al. Heterogeneity of EGFR-mutant clones and PD-L1 highly expressing clones affects treatment efficacy of EGFR-TKI and PD-1 inhibitor. Ann Oncol 2018;29:2145-7.

27. Testa U, Castelli G, Pelosi E. Lung Cancers: Molecular Characterization, Clonal Heterogeneity and Evolution, and Cancer Stem Cells. Cancers (Basel) 2018. doi: 10.3390/cancers 10080248 . 